\title{
Interpretation of the Beauty of Contemporary Digital Fashion Paintings
}

\author{
Yuexia Yao \\ Silicon Lake College \\ Kunshan, Jiangsu, China 215332
}

\begin{abstract}
In many contemporary art forms, there is a kind of painting form with modern multimedia technology as carrier. It is a product of the perfect combination of clothing art and computer technology. It is the most important technical and artistic activity to form the finished product of clothing link. It is also the most scientific presentation of artistic fashion design and original works. It has a unique aesthetic and practical value. From the view of art, a good digital fashion painting should highlight the expression of its artistry and beauty in painting works. For a designer's works, the designer may pay more attention to the aesthetics of technology when he/she designs with a computer. Most often the artistic beauty and the technological beauty could be presented in same works.
\end{abstract}

\section{Keywords - appreciation; digital fashion painting; the beauty}

\section{INTRODUCTION}

With contemporary multimedia technology as platform, digital fashion painting is a type of painting form, different to traditional hand-painted fashion painting. It shoulders the responsibility of the most "original" medium to express designers" creativity and ideas. It is a silent "language" for designers to carry out artistic creation and make unique innovations, and a "visual impact" on future customers to select favorite clothing commodities. The digital fashion paintings have more era character and technological character than that of hand-painted paintings. It could better present designers' ideas and concepts intuitively and quickly, so more and more people begin to use it. Continuously innovative "artistic beauty" is a dominant characteristic of digital fashion paintings all the time. The implicit "technological beauty" is often ignored by people. The paper is intended to attract people's attention on the long existing technological beauty of digital fashion painting and help people have a correct understanding on the relationship of artistic beauty and technological beauty by analyzing the digital fashion paintings. The discussed scope of digital fashion paintings refers to the designs by using computer aided technology for later development, research, production and sales.

\section{THE EMERGENCE AND DEVELOPMENT OF DigITAL FASHION PAINTING}

Fashion painting appeared in the middle of 16th Century. British Wenceslaus Hollar painted the first clothing painting of fashionable effect with etching method in the world [1]. From that time till 1930s, the fashion painting had been the advertisement of the new clothes. It often appeared in popular magazines, newspapers and in the window of clothing store, facing the public to disseminate fashion beauty. However, starting from the end of the 1930s, with the rise of techniques for photography, film image gradually substituted as the medium to advertise new clothes, and fashion painting gradually receded from people's sight. But it turned from the characteristic of "propaganda" to the characteristic of "function" in fashion design gradually. Fashion painting, with fashionable clothes as subject, is to show the image effect of the clothes after people dress it. It is the embodiment of creative inspiration of fashion designer, and a "dominant business card" to connect designer, craftsman and consumers. It has a strong artistic aesthetic character and technological practicability.

The emergence of digital fashion paintings is by the aid of continuously updated computer aided design technology. It is a new painting form in the fashion field, and a highly comprehensive discipline. Its existence has brought a profound change to clothing teaching and clothing design market. Digital fashion painting is based on the Computer Aided Design (referred to as CAD) technology. The professional software used includes garment computer aided design software and the general graphic design software, such as Photoshop, Illustrator, CorelDraw, Painter and so on. Based on the convenient, fast presentation and other advantages, now more and more clothing colleges and universities and designers are willing to abandon the pure hand-painted painting and select digital fashion painting to present the design ideas and concepts, to meet today's needs and trends in fashion design.

Only by deeply understanding its advantage, can you understand and feel the technological and artistic beauty contained in digital fashion paintings. Compared with pure hand-painted fashion paintings, the digital fashion painting has the following advantages:

\section{A. The Diversity of Presentation Tools and Techniques}

With powerful editing, modification, storage and other functions of the digital paint software, and the setting of various parameters, designers can easily imitate the brushwork of traditional painting, and even easily achieve incomparable visual image that hand-painted painting could not match. Designers could easily get the wanted effects in the color of the deployment, line changes, the tone of shade, the gradation 
of layers, the shaping of the texture, simulation of light and so on.

\section{B. The Fast and Efficient Creative Process}

Compared with pure hand-painted fashion paintings, the digital fashion painting can be modified and recombined repeatedly and pre-presented with the aid of computer software and in-time dominant characteristic. The preset effect could be finished according to designers' ideas and concepts in first time. The processes, including sketch design and coloring, depiction of fabric texture, selection and design of pattern and color matching of entire garment, can be presented quickly through computer, for customer selection and determination.

\section{The Secure and Powerful Storage and Reuse Advantage}

It benefits from the powerful memory and storage function of computer. The creation process of fashion painting can be stored one step at a time, which is convenient for future modification and reuse. And it takes up a little room. The finished design product can be transmitted remotely through Internet technology, and printed, or disseminated and spread by micro media and new media around the world.

\section{THE REPRESENTATION FORM OF THE BEAUTY OF DIGITAL FASHION PAINTINGS}

The said artistic beauty, namely the beauty of art works, is a kind of beauty that is created and produced according to aesthetic understanding of artists and the law of beauty for the purpose of beauty appreciation. To create the artistic beauty is the fundamental purpose and the highest requirement for artists, and the ultimate accomplishment of artists in art [2]. The artistic beauty of digital fashion paintings is the result that is produced according to aesthetic understanding of fashion designers and the law of beauty. Whether to represent conceptual clothing or practical clothing, the design thinking of designers is inseparable to the beautiful design and creation in creating digital fashion paintings. In addition to the representation of the wholeness, figurativeness, emotional sense, individuality of the artistic beauty [3], the design works should highlight the artistic beauty of digital fashion paintings. It can be summarized in the following features:

\section{A. The Beauty of Composition}

Before conceiving and painting, the designer shall have the design work in mind. It involves in the dressing figure and the composition style and form of the clothes. Clothes are specific commodities, and ultimately are made for sale, but any plain design cannot be inseparable with creativity and imagination. As a designer, you can't give up the original intention on innovative clothing, and shall achieve the best dressing effect with well-controlled picture form.

\section{B. The Beauty of Color}

For any piece of clothing, the first to attract people's eyes and give visual stimulation is the color. The color layers shall have the sense of gradation, rhythmic beauty, and sense of rhythm. Color matching shall not only reflect the "soul" of different styles of clothing, but also pay more attention to the visual pleasure and psychological satisfaction that the color could bring to the wearer. Clothing materials are closely related to fashion colors. The selection, design and expression of garment material and accessories should take the enjoyment that clothing texture effect has into consideration.

\section{The Beauty of Integral Matching}

In creating any fashion painting, the final presentation effect should be integral, and have shining point to attract viewers. The integral and harmonious beauty of fashion painting shall be presented in design concept, creation of style, color matching, selection of fabric, embellishment of accessories, and grasp of the overall style.

\section{The Aesthetics of TeChNOLOGy PRINCIPLE OF DIGITAL FASHION PAINTING}

The aesthetics of technology is the technical performance of researching aesthetic consciousness. It is a science with appropriate combination of human's spiritual needs and material needs, and a branch of applied aesthetics. It is also a comprehensive discipline of technological science and aesthetics [4].

The aesthetics of technology has been accompanied by people's life and has played a more and more important role from handicraft industry age to the industrial age, and till today's information era. The application traces of technological beauty can be seen in all aspects of people's basic necessities. With digital fashion painting as carrier, the paper has analyzed the technological beauty embodied in the creation of digital fashion paintings. The Beauty of Technology Scholar Xu Hengchun has pointed out in "The Aesthetics of Technology": "the beauty of the product with united practical and aesthetic function produced in creative production work is the technological beauty. It is the first form of beauty in human's creation, and also the most basic aesthetic existence in human's material life"[5]. In other words, the practicability (functionality) is the first feature of the technological beauty. On the basis of this, the beauty of form of product should also be taken into consideration to achieve the double orientation of function and aesthetic appreciation, and psychological pleasure. As for clothes with commercialization as main feature, its design and production processes have always run through and reflect the aesthetic appreciation of function and art. Seen from the process of clothing production, the digital fashion painting, as one of original design and representation carriers, is no exception. So in the specific creation process of digital fashion paintings, the functionality of design object, clothing, should be put into important position all along. Some people say that unwearable clothes are waste. It also proved that designers should put wearability, practicality, protection against cold, cover, temperature adjustment and other factors related to ergonomics into first place, so that the creation of fashion design could meet people's basic physiological requirements in technological level. On the basis of this, then consider the beauty of form. In addition, the most important of digital fashion paintings is the fashion style. What's more, it should be based in scientific and technical progress. Take a suit jacket painting for example. The style and outline of the suit shall be 
determined according to related data, appropriate proportion of human body and the provided size. The parts, including collar type, shoulders, sleeves, pockets and flaps, should be proportional, coordinated and accurate. Every detail should be covered in graphic design, and the processing of material texture. From pin mark, density of stitch to pattern of whole garment, all details could reflect an accurate and professional design.

The technological beauty of digital fashion paintings has existed for a long time, because any creative works is inseparable from technical activity besides the beauty of artistic form. The technological beauty is the spiritual crystallization of human activities. At this point, the technological beauty is closely linked to functions of digital fashion paintings and the representation of clothing. It is premised on "usefulness". The discussed scope of digital fashion paintings refers to the designs by using computer aided technology for later development, research, production and sales. Hence, it emphasizes whether the originally developed product could be used for future sale. It is company's purpose, design needs, and industrial rules. The technological requirements and aesthetic grasp should be the key in digital fashion paintings. The technical requirements constantly run through the entire activity, which is also verified that the beauty of technology is the aesthetic value orientation with united "content and form".

\section{THE RELATIONSHIP OF THE ARTISTIC BEAUTY AND THE TECHNOLOGICAL BEAUTY OF DigITAL FASHION PAINTINGS}

In order to clarify the relationship of the artistic beauty and the technological beauty of digital fashion paintings, we should first understand the relationship of art and technology in the field of aesthetics. In the opposition theory of "art and technology", it is generally thought that the fundamental purpose of art is to create the value of beauty, and the fundament purpose of technology is to create an actual utility [6]. From the point of this view, art and technology have completely different aesthetic orientations and purposes. But one thing is undeniable both art and technology have the behavior of processing materials according their respective purposes. If we call this creation skill of human technology, art, same as technology, could be included in generalized technological scope [7]. That is to say, art and technology are in a compatible relationship and mutually included at some level. Art contains technological character and technological beauty. Technology also is permeated with the beauty of art. Both artistic activities and technological activities cannot be separated from human's activities in creation of beauty. Technology and art have been united as indivisible entirety since the period of Ancient Greece. Both of them are contained in the concept of "technology". The technological beauty and artistic beauty are jointly produced in the product design and production process.

The creation of digital fashion paintings is also a kind of intelligent activity, which depends on the production of commercial clothing that could meet the atheistic value of the public and are acceptable to the public. With the mission of disseminating beauty, the fashion creation integrates both artistry and technicality. Both are indispensable. In the atheistic principle, fashion design has two types of atheistic standards. One takes originality as beauty, and the other takes practicality as beauty. Therefore, with different atheistic standards, the creation purposes and requirements of digital fashion paintings are also different. To be exact, according to different aesthetic principles and design principles, the requirements and grasp of artistic beauty and technological beauty are not the same. For example, according to the aesthetic principle of originality, the digital fashion painting shall highlight artistry and innovation, and stress the artistic beauty in style, color, modeling, matching and other aspects to be "attractable" and "unique". For this, the status and requirements of technological beauty cannot be dominated, but give way to the artistic beauty. Of course, it is not to say that the status of technological beauty completely disappeared at this moment. It just does not hold the "leading" position. Similarly, if taking the design principle of practical beauty, it shall stress the form of clothing, exquisite technological design and production and other practical and functional characters in order to meet the atheistic value and demands of the public.

\section{CONCLUSION}

Digital fashion painting is a product of the perfect combination of art and technology. From conception to specific styles, colors, fabrics allocation and other detail treatments, all of them show the beautiful image in graphic design sketch. For the originator and recipient of digital fashion paintings, whether it is the pursuit of technical beauty or artistic beauty, their forms and contents are unified and accrete. Only by knowing this point, would people not take digital fashion painting only as "watchable" ornament, for it contains highly technological beauty.

\section{REFERENCES}

[1] Tian Meiling. Interpretation of the Era Character of Fashion Painting. Ornament, 2005 (7).

[2] Yu Yongshun. Introduction to the Beauty of Art. Shenyang: Liaohai Press, 2006.

[3] Xie Yijia. Research of Artistic and Technological Beauty in Virtual Reality System. Shandong University, 2009.

[4] Zhong Hua. Influence of the Aesthetics of Technology on Product Design. Culture and Education Materials, 2005 (32).

[5] Xu Hengchun. The Aesthetics of Technology. Shanghai: Shanghai People's Publishing House, 1989:1 10.

[6] Takeuti Toshio, Leshan. - Art · Technology • Beauty. Journal of Nanjing Arts Institute (Art and Design Edition), 1990 (2). 